\title{
CONOCIMIENTO DE LAS ENFERMEDADES UROGENITALES EN LA OBRA DE GASPAR CASAL, "HISTORIA NATURAL Y MEDICA DEL PRINCIPADO DE ASTURIAS" (1762)
}

\author{
Luis Ángel Fariña Pérez.
}

Servicio de Urología. Hospital Povisa. Vigo. Pontevedra. España.

\begin{abstract}
Resumen.- OBJETIVO: Gaspar Casal (Gerona 1680, Madrid 1759) realiza la mayor parte de su obra médica en Oviedo, donde vive treinta y cuatro años, antes de volver a Madrid como médico de la Real Cámara. Fruto de su trabajo en Asturias es el libro póstumo "Historia natural y médica del Principado de Asturias" (Madrid, 1762), considerado el mejor exponente de la medicina española del siglo XVIII, y donde se describe por primera vez el "mal de la rosa", más tarde llamada pelagra o avitaminosis B.
\end{abstract}

MÉTODOS: Estudio de la vida de Gaspar Casal y análisis de su única obra "Historia natural y médica del Principado de Asturias", especulando sobre el conocimiento de las enfermedades nefro-urológicas y genitales que se deduce de este texto.

\author{
Luis Ángel Fariña \\ Servicio de Urología \\ Policlínico Povisa \\ Salamanca, 5 \\ 36211 Vigo. Pontevedra. (España). \\ luisfarina@yahoo.com
}

RESULTADOS: Casal conoce y trata la litiasis renal, que relaciona en unas ocasiones con las dolencias articulares y la gota, y en otras con la abundancia de comida y la vida sedentaria. Otras enfermedades acompañadas de poliuria y edemas que describe, son compatibles con el diagnóstico actual de nefritis infecciosa o degenerativa. Utiliza en pocas ocasiones el sondaje vesical en los casos de estranguria y anuria, incluye posiblemente en el término de "iscuria renal o supresión alta" a algunos enfermos con anuria y a otros con retención de orina, aunque también usa el término "iscuria vesical" para estos últimos. Los sintomas disuria-estranguria y hematuria, los trata sólo con terapia empírica, y se intuye en su texto que para los cirujanos locales no era habitual el alivio de estos sintomas mediante el sondaje uretral. Atiende una epidemia de paperas, con orquitis en los varones, que trata mediante sangrías, aunque se mostró contrario al abuso de las mismas. Sólo ocasionalmente hace tratar quirúrgicamente ciertas enfermedades testiculares.

CONCLUSIONES: "Historia natural y médica del Principado de Asturias" refleja el espectro de las enfermedades que en la primera mitad del siglo XVIII ocupaban el trabajo de un médico, a saber, las derivadas de las carencias alimentarias, infecciosas, parasitarias y las degenerativas y vásculo-cerebrales en personas añosas. Desde el punto de vista nefro-urológico, es muy interesante comprobar el conocimiento en esa época de la enfermedad litiásica y de los síntomas del tramo urinario inferior, tratados al modo recomendado por los clásicos, con tratamiento físico y preparados empíricos, pocas veces con sondaje; y, en menor medida, los resultados del tratamiento médico de las orquitis y el tratamiento quirúrgico de algunas lesiones testiculares.

Palabras clave: Gaspar Casal. Principado Asturias. Enfermedades urogenitales. Historia de la Urología. 
Summary.- OBJECTIVES: Gaspar Casal (Gerona, 1680, Madrid, 1759) made most of his medical work in Oviedo (Asturias, Spain), where he lived for thirty-four years, before his return to Madrid as a doctor for the Royal House. Fruits of this work is the book "Natural and medical history of the Principality of Asturias" Madrid, 17621, considered the best exponent of the Spanish medicine of the 18th century, and where the "disease of the rose", known later as pellagra or hypovitaminosis B, was first described in.

METHODS: Study of the life of Gaspar Casal and analysis of "Natural and medical history of the Principality of Asturias", speculating on the knowledge about nephro-urologic and external genitalia diseases that can be deduced from the text.

RESULTS: Casal knows and treats kidney lithiasis, relating it either to joint diseases and gout, or to dietetic excess and sedentary life in some of his patients. Other diseases coursing with poliuria and oedemas, are compatible with the nowadays diagnosis of infectious or degenerative nephritis. He rarely uses a bladder catheter for cases of stranguria and anuria, probably including some patients with anuria and others with urinary retention in the term "renal ischuria or high suppression", although he also uses the term "vesical ischuria" for the last ones. He treats the symptoms disuria-stranguria and haematuria, with empiric therapy, and, as it may be deduced from the text, some local surgeons didn't use or know to alleviate those symptoms with urethral catheterisation. He attends an epidemics of mumps, with orchitis in male patients, that were treated with bloodletting, although he stood against the misuse of phlebotomy in several diseases. Only occasionally he orders surgical treatment for several testis diseases.

CONCLUSIONS: "Natural and medical history of the Principality of Asturias" reflects the spectrum of diseases that conforms the day-to-day working schedule of a Spanish family doctor in the beginning of the 18th century, that is to say: diseases resulting from dietary shortage, infective, parasite, degenerative and vascular-cerebral diseases in older patients. From the nephrourologic point of view, it is very interesting to read about the knowledge on stone diseases and lower urinary tract symptoms, that were treated as recommended by the classic authors, with physical therapy and empiric formulas, rarely with bladder catheterization. To a lesser degree, the results of the medical treatment of orchitis and the surgical treatment of some testis diseases are also depicted.

Keywords: Gaspar Casal. Principality of Asturias. Urogenital diseases. History of Urology.

\section{INTRODUCCIÓN}

Nacido en Gerona en 1680 y formado tal vez en las Universidades de Sigüenza y Alcalá de Henares, aunque no se conocen documentos que prueben su formación académica, Gaspar Casal comienza su ejercicio profesional en Madrid en 1713, atendiendo a una clientela distinguida. Se traslada en 1717 a Oviedo, donde durante treinta y cuatro años realiza la mayor parte de su vida profesional, razón por la que se le considera hijo de esta ciudad, primero como médico al servicio del ayuntamiento y luego del Cabildo de la Catedral. Su fama se extendió pronto, se hizo cargo de la dirección de varios de los pequeños hospitales del Oviedo de entonces, que tenía unos 6.000 habitantes (hospitales de Santiago, San Juan y Santa María de los Remedios, actualmente desaparecidos) (1,2), (Figura 1), y visitaba a enfermos de todo el principado y de las provincias vecinas. En 1751 regresa a Madrid como médico de la Real Cámara y miembro del tribunal del Real Protomedicato, y donde es elegido miembro de la Real Academia de Medicina. Falleció en 1759 y fue enterrado en Madrid, sin que se conozca ninguna imagen de su fisonomía.

Fruto de su trabajo en Asturias es el libro, "Historia natural y médica del Principado de Asturias", publicación póstuma que se imprime en Ma-



FIGURA 1. Plano de la ciudad de Oviedo de Francisco Reiter (1777), algunos años después de la estancia de Casal. Tomado de Tolivar (5). 
drid, en 1762, por decisión de su compañero del protomedicato, el Dr. Juan José Garcia Servillano (Figura 2). Este libro, que recoge seis textos diferentes de Casal, está considerado el mejor exponente de la medicina española del siglo XVIII, con relato de historias clínicas y exposiciones basadas en las observaciones del autor. En uno de los textos se describe por primera vez el "mal de la rosa", primer estudio clínico de una enfermedad carencial, más tarde llamada pelagra o avitaminosis $B$, con un signo característico que se conocerá como "collar de Casal" en la bibliografía universal.

El libro se reeditó en el año 1884, en los años 1900 y 1959, estas dos últimas con prólogos de Angel Pulido y de Gregorio Marañón y, más recientemente, en el año 1988 (3), con una introducción del Dr. Tolivar, gran conocedor de la vida y de la obra de Casal $(4,5)$. Esta edición facsimilar de la principal, incluye una traducción al castellano de algunos capítulos que fueron escritos en latín. El contenido de este texto ha sido objeto de varios análisis

HISTORIA

NATURAL, Y MEDICA

DE EL PRINCIPADO DE ASTURIAS.

OBRA POSTHUMA,

QUE ESCRIBIO' EL DOCT. D. GASPAR

Cafál , Medico de fu Mageftad, y fu Proto.

Medico de Caftilla, Academico de la

Real Academia Medica

Matritenfe,\&c.

LA SACA A LUZ

EL DOCT. JUAN JOSEPH GARCIA Sevillano, Medico de Familia del Rey nueftro Seńor, Ex-Examinador de fu Real Proto-Medicato, Medico que ha fido de los Reales Hofpitales, y actual de el Real Sitio de Buen Retiro, Academico de la Real Academia Medica Matritenfe, y de la Real de Oporto, \&c.

CON LICENCIA : En Madrid, en la Oficina de Manuel Martin, Calle de la Cruz. Ańo de 1762 .

Se ballarà en la Libreria de Don Francifgo Manuel de Mens, Calle de las Carretas.

FIGURA 2. Portada del libro de Gaspar Casal. historiográficos, desde el punto de vista de la cardiología, la neurología y la pediatría (6-8), además de los extensos estudios que analizan la principal aportación de Casal, la descripción del mal de la rosa - lepra asturiense (pelagra) $(9,10)$, pero aún no se había estudiado desde el punto de vista del conocimiento de las enfermedades nefro-urológicas y de los genitales que refleja.

\section{MATERIAL Y MÉTODO}

Estudio de la vida de Gaspar Casal y análisis de su única obra "Historia natural y médica del Principado de Asturias", en la edición facsimil de la principal, del año 1988, especulando sobre el conocimiento de las enfermedades nefro-urológicas y genitales que se deduce de este texto.

\section{RESULTADOS}

Casal señala a los cálculos de los riñones y vejiga entre las indisposiciones propias del pais, "endémicas". La litiasis renal, única dolencia urológica a la que dedica un capítulo, en el primero de los textos, la considera un achaque frecuente ("de los moradores que comen, beben vino y trabajan poco, son muy singulares los que no los padecen"), y no se para en describir los síntomas, por considerar que "ya los autores prácticos lo dejaron harto bien delineado". Confirma su relación con la abundancia de comida y la vida sedentaria, pues era enfermedad propia de "ricos y ociosos" y no de labradores ("andan llenos de males los ricos y los pobres: aquellos, porque no trabajan según comen y beben, y éstos, porque no beben ni comen según trabajan"). También recuerda la asociación entre los cálculos y los dolores artríticos y la gota, señalando que los gotosos suelen tener cólicos, pero por el contrario, los "nefríticos" no siempre son gotosos, basándose para esto en una relación de 22 pacientes litiásicos conocidos, que no son gotosos o artríticos.

Describe tres pacientes para ilustrar esta dolencia, el primero, con una crisis de cólico agudo, fue tratado con aceite de almendras dulces y jarabe de adormideras, expulsando a las cinco horas "tres piedrecitas casi como garbanzos". El segundo y el tercer caso son pacientes más complejos, con antecedentes de cálculos, pero con episodios de retención de orina, infección febril y otras complicaciones, que le sirven a Casal para manifestarse contra los tratamientos intensivos que los médicos y cirujanos que le precedieron en la consulta a los pacientes, practicaban, a base de purgantes y diuréticos potentes, con complicaciones dramáticas. Por el contrario, obser- 
va que los paciente que se dejaban "a beneficio de la naturaleza", sin ayuda médica, "sólo con caldos, agua tibia, ayudas y unturas emolientes", eliminan también piedras (Figura 3). También se muestra contrario al uso de la sangría, salvo en casos especiales.

Algunos de los enfermos que describe en otros apartados, con enfermedad acompañada de poliuria y edemas, parecen tener cuadros compatibles con el diagnóstico actual de nefritis infecciosa o degenerativa, muchas veces consecuencia de obstrucción infravesical o de cálculos renales o vesicales complicados. Se vale de la inspección en orinal de vidrio de las características de la orina y los cambios de su aspecto con la sedimentación para, en estas enfermedades y en casos de fiebres epidémicas, sacar algunas conclusiones sobre la naturaleza de la enfermedad y su pronóstico "aunque en las fiebre malignas suelen no ser constantes las reglas y principios generales".

No quiero pintar el modo, y fymptomas, con que acontecen en efte Pais los infultos, ò paroxifmos nephriticos: porque (facando aquellas individuales difezencias, que en todo mal fe perciben) yz̀ los Autores práticos lo dexaron harto bien delineado. Solo debo decir, que, como en Afturias abundan tanto los cuerpos de cradezas, y fuperfluitdades, no fe puede tan prontamente ufar de la evacuacion de fingre, como en otras Regiones; à no fer, que la plenitud, juventud, robuftèz, edad, y otras particulares difpoficiones de los pacientes la pidan

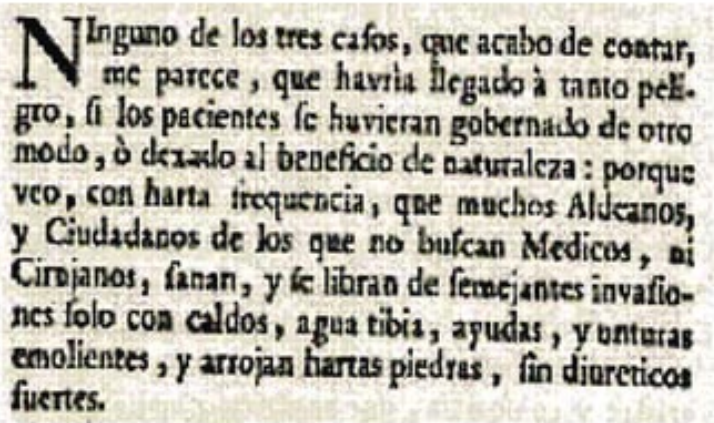

FIGURA 3. Referencias en el capítulo dedicado a los cálculos renales, a la conocida sintomatología clínica de estos episodios, y lamento por los tratamientos intensos que reciben los pacientes en ocasiones, en una enfermedad que Casal considera de curso frecuentemente benigno.
Los síntomas vesicales los considera muy frecuentes, ("en este país son tan frecuentes las disurias, estrangurias $y$, en los viejos, los repetidos estímulos de la orina que especialmente de noche, les interrumpen el sueño"), si bien no identifica a la uretra o la próstata como posible origen de enfermedad. Con respeto al tratamiento, confiesa que "he procurado tentar y probar cuantas medicinas antiguas y modernas, simples y compuestas, pude hallar escritas en los autores de mejor nota y más selectas farmacopeas, para corregir semejantes irritaciones". Finalmente el mejor tratamiento que conoce son los "polvos de erizo entero, quemado y calcinado, hasta reducirse a cal o ceniza blanca", asociado a emulsiones de pepitas variadas, cocimientos o infusiones de plantas diversas. En la exposición de estos casos, que ejemplifica con algunos pacientes cuyo sufrimiento vesical está relatado de manera muy explícita ("las orinas, en algunas ocasiones, venían con tan excesivos ardores, dolores y tormentos, que para expeler cualquier corta porción, gritaban como locos, encorvando el cuerpo y haciendo extraordinarios movimiento"), incluye posiblemente en el término de "iscuria renal o supresión alta" a algunos enfermos con anuria y a otros con retención de orina, no es posible dilucidar esta información por los datos que da, aunque aclara que la estranguria daría lugar a la iscuria, y también usa el término "iscuria vesical o supresión baja" para estos últimos, cuadro del que dice "tengo vistas muchísimas... estas supresiones son muy manifiestas, y a primera vista se conocen en los dolores, peso, ardor, tensión y tumefacción de la región que ocupa la vejiga".

En una extensa disertación, pretende aclarar la naturaleza de los síntomas abdominales que siguen a la estranguria no tratada, que, unos llamaron ileo o pasión ilíaca y otros vólvulo intestinal y que, según los tratados clásicos, llevan siempre a la muerte del enfermo "en el espacio de siete dias", argumento contra el que expone su larga experiencia y la imposibilidad de recordar un solo caso de esta evolución ("nunca pude hallar observación escrita de semejante mudanza... y en verdad que si tal mutación fuera regular, como otras, creo que la habría hallado"). En una de las observaciones con las que quiere ilustrar esta afirmación, relata: "Pretendí averiguar si en la vejiga se había juntado alguna porción de orina: y tomando la sonda, pues sabía manejarla, la introduje hasta el fondo mismo de la vejiga, sin ocasionar la más leve molestia al enfermo, pero fue en vano este registro, porque no encontré más que tres o cuatro gotas". No obstante, se intuye en el texto que ni para Casal ni para los cirujanos locales que en ocasiones le precedían en las consultas, era habitual la identificación y el alivio de estos síntomas vesicales mediante el sondaje uretral, una técnica que 
para entonces pocos dominaban. Así, señala que las supresiones bajas se curaban "con fomentos, baños, enemas específicas, emulsiones diuréticas benignas, sangrías en sujetos pletóricos, y en casos apurados con el instrumento que llaman algalia".

En los años 1720 y 1747 atiende epidemias de paperas, en el último año, con la particularidad de que "fue mayor el número de los que padecieron el decúbito a los testes". En estos casos optó por la sangría, "el único y seguro remedio, que desterraba la calentura, y minoraba la inflamación dolorosa; y así los que se sangraban, conseguían presto la salud, haciéndoles después algunos oportunos fomentos sobre el teste entumecido" sin encontrar fallecimientos entre los pacientes.

Las lesiones de la región inguinal y genital, incluyendo hernias y lesiones intraescrotales no fueron bien conocidas de Casal, aunque identificara bien las "hernias intestinales que llaman quebraduras". Sólo ocasionalmente hace tratar quirúrgicamente ciertas enfermedades testiculares, que en algún caso parecen corresponder a orquitis tuberculosa o a tumor de teste con metástasis pulmonares. Uno de los pacientes, un joven robusto, "comenzó a padecer una tos seca, que le repetía con frecuencia. Persistiendo esta tos por largo tiempo, se fue entumeciendo el testículo izquierdo, hasta formarse una hernia del tamaño de un huevo de gallina, con señas de carnosa". Fue intervenido por cirujanos y falleció "de una fatal gangrena". Otro varón joven, comenzó con tos y fiebre, sobre todo vespertina, que mejoró después de que apareciera tumefacción del testículo izquierdo. Fue operado por el cirujano francés Juan Delgart, "con lo cual quedó el paciente perfectamente curado".

Casal hizo las primeras aplicacione en Oviedo de mercurio para el tratamiento de casos diagnosticados de lepra y sífilis, también lo usó en un niño de quince años con lesiones que ahora podríamos identificar como quistes sebáceos múltiples o calcinosis de escroto. Los tumores urogenitales, con excepción del caso comentado, no aparecen en la obra de Casal, pero el ultimo de los textos del libro es la descripción de un probable caso de cáncer de mama en un varón, la más larga de las historias clínicas de Casal, con la evolución natural de la enfermedad, tratada con unciones mercuriales que lógicamente no cambiaron su curso, con aparición de úlcera, adenopatías, metástasis óseas y fracturas patológicas En este caso, Casal consultó con "los sapientísimos doctores de Paris", entre los que se encontraba el cirujano Jean Louis Petit, uno de los fundadores de la medicina anátomo-clínica, y anota los consejos recibidos y el resultado de su aplicación.

\section{COMENTARIO}

Dentro de los periodos temporales y corrientes de pensamiento en que se divide con intención académica la historia de la medicina en Europa, la primera mitad del siglo XVIII en la que Casal desarrolla su vida médica, corresponde al periodo final de la transición de la "medicina renacentista" a la llamada "primera medicina moderna". En esta épo$\mathrm{ca}$, si bien sigue prevaleciendo la doctrina griega de la enfermedad, transmitida en los escritos de Hipócrates, Galeno y los médicos árabes, están teniendo lugar los cambios ideológicos y técnicos que inducirán la orientación anatómica y fisiológica que un siglo después llevarán a la concepción de la medicina contemporánea, que interpretará las enfermedades con un sustrato lesional particular para cada órgano $(11,12)$. A este hecho no es ajena la difusión de la imprenta, que permite una difusión más rápida y barata de las nuevas traducciones de los clásicos, y de textos originales (13), escritos con un espíritu crítico en diferentes países europeos, y que tenían en común un aspecto novedoso: incluían reflexiones sobre la experiencia personal de los autores. Tampoco lo es el interés que se presta a las enfermedades infecciosas y carenciales que, sin ser nuevas, afectan ahora a sectores crecientes de la población $(14,15)$.

El contexto español de la época, se caracteriza por la guerra de Sucesión al trono (1702-1713), y el cambio de la dinastía de los Austria por la de los Borbón, con el largo reinado de Felipe $\mathrm{V}$, nieto de Luis XIV de Francia. Un momento caótico en lo económico, con la industria arruinada y la población diezmada por las epidemias y las guerras y por lo general sumida en la superstición y la ignorancia, y en el que sólo las órdenes religiosas mantienen su riqueza y su expansión (16). A lo largo del siglo, la influencia de los dos imperios que gobiernan Europa central, Francia y Austria, y las ideas de la llustración, se sentirán en la ciencia y medicina española, entre otras cosas con la llegada de médicos y cirujanos del ejército francés, capaces de realizar intervenciones de mayor envergadura, como amputaciones y herniotomías, y otras incluyendo los sondajes y la litotomía (17).

Aunque el ejercicio de la medicina en tiempos de Casal estaba condicionada a la demostración de una capacitación suficiente ante el Protomedicato, no se puede obviar que prevalecía la formación universitaria, en latín y muchas veces ajena a los cambios que se estaban produciendo en todos los órdenes, ni tampoco la influencia de la lglesia, que exigía para la práctica médica la pureza de sangre y el juramento a la defensa del misterio de la Concepción (1). La formación de los cirujanos era mucho más pobre, en ocasiones no mucho mayor que la de otros 
profesionales que formaban parte de la profesión sanitaria como los barberos y los sangradores.

Los textos de Casal reflejan lo que era la clínica en su tiempo. En su constante identificación de los enfermos, con nombres y relaciones de parentesco, en particular si eran de familias conocidas, y en su repetida afirmación de que dice la verdad, se detecta la obsesión por mostrar que no miente y diferenciarse así de lo que parecía una practica común entonces, con objeto de procurarse la clientela (5). Sus enfermos son de toda condición social, aunque destacan los enfermos notables dentro de la profesión eclesial. El libro está repleto de referencias a su experiencia personal, - "soy fino amante de la experiencia, y enemigo capital de aquellas fruslerías mentales fundadas sobre hipótesis y dogmas", dice-, y a sus conocimientos de los textos médicos de la época, que a veces contrapone al criterio que considera poco formado de otros médicos de su área geográfica.

Aunque no se han encontrado documentos que prueben su formación médica, su amplísimo ámbito de referencias muestran una destacada bibliofilia y profunda ambición de conocimientos (5). Así, podemos comprobar que hace referencia, muchas veces para rebatirlos, sobre todo a Hipócrates y los médicos que en el siglo XVI divulgaron los tratados hipocráticos, y el saber griego y arábigo, Louis Duret, Juan Marinelli y Jean Fernel. También a Celso y a Plinio (siglo I), cuya "Historia Natural" fue traducida al castellano a comienzos del siglo XVII, a Galeno y a su contemporáneo Marciano (siglo II), que comentó las obras de Hipócrates.

Más modernas, entre sus variadas fuentes de información, que abarcan más de 40 diversos nombres, encontramos citas de contemporáneos o que publicaron obras importantes en los años precedentes, desde el italiano Giorgio Baglivi (16681707), que enseñó en Roma, los alemanes Johann Baptista van Helmont (1577-1644), Federico Hoffman (1660-1742), Michael Ettmüller (1644-1683) y el holandés Herman Boerhaave (1668-1738), quienes habían establecido formas nuevas de interpretar la enfermedad (iatromecanica y iatroquimica), diferentes a la interpretación humoral del galenismo; hasta Francis Bacon (1561-1626) y Thomas Sydenham (1624-1689), quien con su concepto de especie morbosa fue uno de los primeros en intentar establecer unas formas típicas de enfermar, y contribuyó significativamente a clasificar las enfermedades humanas, al modo en que Linneo ordenaba por entonces las plantas, pero basándose en los síntomas, la personalidad del paciente y la época del año $(11,12,18)$. También hace numerosas citas de autores de textos de química y farmacopea. Entre los autores españoles que en esos años ejercieron influencia académica, destaca al "doctísimo, honra de los médicos españoles" Francisco Vallés (1524-1592), catedrático de Alcalá y médico de Felipe II, que escribió comentarios a los libros hipocráticos y de Galeno, a Martín Martínez (16841734), importante médico de su tiempo y autor de una influyente "Anatomía completa" (19), y al sevillano Nicolás Monardes (1507-1588), del que Casal hace referencia a su obra "Historia medicinal de las cosas que se traen de nuestras Indias Occidentales".

Es muy destacable su amistad con los frailes benedictinos Benito Feijóo y Martín Sarmiento, sabios ilustrados que sin ser médicos, escribieron textos criticando la medicina empírica de su tiempo, y urgieron a la práctica de una medicina fundamentada en la experiencia y en el uso de medicamentos cuyos efectos se hubieran comprobado. Casal no referencia obras que hoy consideramos tienen un gran interés desde el punto de vista del conocimiento de las enfermedades urogenitales, como son los escritos atribuidos a Julián Gutiérrez de Toledo, y los de Andrés Laguna, Francisco Díaz o Miguel de Leriza, ni de otros tratadistas médicos como Luis Mercado, o quirúrgicos como Daza Chacón o Juan Fragoso (20). Se podría echar en falta la existencia de referencias quirúrgicas, pero para entonces los estudios médicos y quirúrgicos estaban muy separados, y los cambios más importantes en la interpretación de la enfermedad quirúrgica ocurrirán años después, tanto en España (Gimbernat), como en Francia (Petit, Chopart y Desault), en Italia (Scarpa) o Inglaterra (Pott, Hunter).

El estudio de la época de Casal muestra con claridad la escasez de cirujanos que había entonces, que eran muy poco considerados y mal pagados, y rara vez pasaban de hacer cuidados de heridas y fracturas, o de atender a los enfermos pobres, mientras el médico cuidaba de las gentes de buena posición (1). Destacan en este sentido en Oviedo dos cirujanos que proceden de los ejércitos de Francia: Juan Delgart, alabado por Feijóo y a quien Casal considera "perito cirujano francés, diestrísimo disector", que también enseñó y practicó cirugía en el Hospital Real de Santiago de Compostela; y Domingo Bucan que disponía de una colección de instrumental médico interesante, que muestra sus habilidades y conocimientos de las técnicas quirúrgicas de cierta complejidad: a su muerte legó este instrumental al Ayuntamiento de Oviedo, y en él se incluían los instrumentos precisos para practicar litotomía, aunque no quedan evidencias de que la pudiera haber hecho en Asturias. 


\section{CONCLUSIÓN}

El texto "Historia natural y médica del Principado de Asturias" (1762), de Gaspar Casal es el mejor ejemplo de la medicina de su tiempo en España y refleja el espectro de las enfermedades que en ese momento ocupaban preferentemente el trabajo de un "médico de familia", a saber, las derivadas de las carencias alimentarias, infecciosas, parasitarias y las degenerativas y vásculo-cerebrales en personas añosas.

Desde el punto de vista nefro-urológico, es muy interesante comprobar el conocimiento en esa época de la enfermedad litiásica y de los síntomas del tramo urinario inferior, tratados al modo recomendado por los clásicos, con tratamiento físico y preparados empíricos; $y$, en menor medida, los resultados del tratamiento médico de las orquitis y el tratamiento quirúrgico de algunas lesiones testiculares.

\section{BIBLIOGRAFIA y LECTURAS RECOMENDADAS (*lectura de interés $y^{* *}$ lectura fundamental)}

1. VILLA, M.P.: "Casal en Oviedo. Estudio documental de los médicos, cirujanos y boticarios de Oviedo en el siglo XVIII". Instituto de Estudios Asturianos, Oviedo, 1967.

2. CABAL, M.: "Hospitales antiguos de Oviedo". Instituto de Estudios Asturianos, Oviedo, 1985.

**3. CASAL, G.: "Historia natural y médica del Principado de Asturias". (Ed. Facs.) Principado de Asturias, Consejeria de Educación, Cultura y Deportes, Oviedo, 1988.

4. TOLIVAR, J.R.: "Introducción”. Casal G., op.cit.

**5. TOLIVAR, J.R.: "Los enfermos de Casal". Instituto de Estudios Asturianos, Oviedo, 1981.
6. BOTAS, V.: "Las enfermedades cardiovasculares en la obra de Gaspar Casal, médico de la ciudad de Oviedo". Rev. Esp. Cardiol., 20: 594, 1967.

7. GARCIA-ALBEA, E.: "La neurología española durante la Ilustración. La obra de Gaspar Casal". Rev. Neurol., 30: 495, 2000.

8. MARTÍNEZ FERNÁNDEZ J.: "Pediatría en las obras de Gaspar Casal". Acta Pediatr. Esp., 19: $45,1961$.

9. LÓPEZ PIÑERO J.M.: "Gaspar Casal: descripción ecológica de la pelagra, primera enfermedad carencial”. Rev. Esp. Salud Publica, 80: 411, 2006.

*10. GARCÍA GUERRA, D.; ALVAREZ ANTUÑA, V.: "Lepra asturiensis. La contribución asturiana en la historia de la pelagra (siglos XVIII-XIX)". Universidad de Oviedo, CSIC., Madrid, 1993.

*11. SÁNCHEZ GRANJEL, L.: "Historia de la medicina". Gráficas Cervantes, Salamanca, 125, 1975.

12. LÓPEZ PIÑERO J.M.: "La medicina en la historia". La esfera de los libros, Madrid, 177, 2002.

13. CLARK, G.: "La Europa moderna 1450-1720". Fondo de Cultura Económica, México, 51, 1986.

14. FAURE O.: "Histoire sociale de la médicine". Anthropos, Paris, 43, 1994.

15. McKEOWN, T.: "Los orígenes de las enfermedades humanas". Triacastela, Madrid, 133, 2006.

16. ELLIOT, J.H.: "La España imperial 1496-1716". Círculo de Lectores, Barcelona, 436, 1996.

17. SÁNCHEZ GRANJEL, L.; RIERA, J.: "Cirugía de la Ilustración. Francia, Italia, España". Laín Entralgo P.: Historia Universal de la Medicina, tomo 5. Ilustración y Romanticismo. Salvat, Barcelona, 126, 1973.

18. BARIETY, M.; COURY, C.H.: "Histoire de la médicine". Librairie Arthème Fayard, Paris, 487, 1963.

19. FÁBREGAS, J.: "El cuerpo humano". Bruguera, Barcelona, 205, 1972.

20. OTERO, I.J.; SERRANO, A.; GOLBANO, J.M. y cols.: "Principales figuras de la urología en el Renacimiento español”. Rev. Urología, 8: 14, 2007 